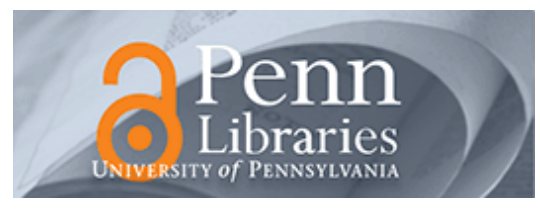

University of Pennsylvania ScholarlyCommons

\title{
The Application of Total Energy as a Lyapunov Function for Mechanical Control Systems
}

Daniel E. Koditschek

University of Pennsylvania, kod@seas.upenn.edu

Follow this and additional works at: https://repository.upenn.edu/ese_papers

Part of the Electrical and Computer Engineering Commons, and the Systems Engineering Commons

\section{Recommended Citation}

Daniel E. Koditschek, "The Application of Total Energy as a Lyapunov Function for Mechanical Control Systems", Contemporary Mathematics 97, 131-157. February 1989.

Preprint version. First published in Control Theory and Multibody Systems, in Volume 97, 1989, pages 131-158, published by the American Mathematical Society in the Contemporary Mathematics series. NOTE: At the time of publication, author Daniel Koditschek was affiliated with Yale University. Currently, he is a faculty member in the Department of Electrical and Systems Engineering at the University of Pennsylvania.

This paper is posted at ScholarlyCommons. https://repository.upenn.edu/ese_papers/672

For more information, please contact repository@pobox.upenn.edu. 


\title{
The Application of Total Energy as a Lyapunov Function for Mechanical Control Systems
}

\author{
Abstract \\ Examination of total energy shows that the global limit behavior of a dissipative mechanical system is \\ essentially equivalent to that of its constituent gradient vector field. The class of "navigation functions" is \\ introduced and shown to result in "almost global" asymptotic stability for closed loop mechanical control \\ systems upon which a navigation function has been imposed as an artifical potential energy. Two \\ examples from the engineering literature - satellite attitude tracking and robot obstacle avoidance - are \\ provided to demonstrate the utility of these observations. \\ For more information: Kod*Lab \\ Disciplines \\ Electrical and Computer Engineering | Engineering | Systems Engineering

\section{Comments} \\ Preprint version. First published in Control Theory and Multibody Systems, in Volume 97, 1989, pages \\ 131-158, published by the American Mathematical Society in the Contemporary Mathematics series. \\ NOTE: At the time of publication, author Daniel Koditschek was affiliated with \\ Yale University. Currently, he is a faculty member in the Department of Electrical \\ and Systems Engineering at the University of Pennsylvania.
}




\section{The Application of Total Energy as a Lyapunov Function for Mechanical Control Systems ${ }^{1}$}

Preprint of a paper to appear in Control Theory and Multibody Systems,

a volume in the American Mathematical Society Series in Contemporary Mathematics .

Daniel E. Koditschek ${ }^{2}$

February 14,1989

1 Keywords: Lyapunov functions, mechanical systems, gradient systems, Hamiltonian systems, robotics.

${ }^{2}$ This work was supported in part by the National Science Foundation under grant no. DMC-8505160 


\begin{abstract}
Examination of total energy shows that the global limit behavior of a dissipative mechanical system is essentially equivalent to that of its constituent gradient vector field. The class of "navigation functions" is introduced and shown to result in "almost global" asymptotic stability for closed loop mechanical control systems upon which a navigation function has been imposed as an artificial potential energy. Two examples from the engineering literature - satellite attitude tracking and robot obstacle avoidance - are provided to demonstrate the utility of these observations.
\end{abstract}




\section{Contents}

1 Introduction 1

2 Dissipative Mechanical Systems 3

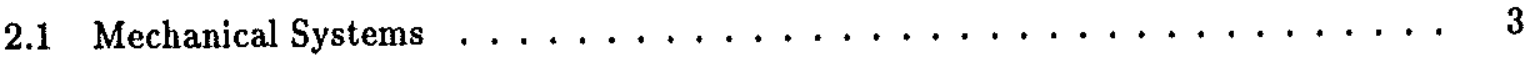

2.1.1 Kinetic Energy as a Riemannian Metric ............... 3

2.1.2 The Mechanical Control System ............... 4

2.2 Gradient Vector Fields in Mechanical Systems . . . . . . . . . . . . 5

2.2.1 Limit Behavior of Gradient Systems $\ldots \ldots \ldots \ldots \ldots$

2.2.2 Lifting Gradient Vector Fields $\ldots \ldots \ldots \ldots \ldots$

2.3 Dissipative Mechanical Systems . . . . . . . . . . . . . 7

2.3.1 Dissipative Vector Fields and Feedback ............. 8

2.3.2 Local Limit Behavior . . . . . . . . . . . . . . . . 8

3 Global Limit Properties of Dissipative Mechanical Systems 9

3.1 Positive Invariance $\ldots \ldots \ldots \ldots \ldots \ldots$

3.1 .1 Rolling Uphill . . . . . . . . . . . . . . . . . 9

3.1.2 Invariance of the Lowest Boundary Energy Set . . . . . . . . . . 10

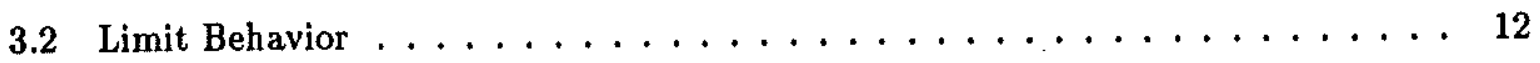

3.2.1 The Limit Set Consists of Equilibrium States . . . . . . . . . . . 12

3.2.2 Local Stability of the Equilibrium States . . . . . . . . . . . 12

3.3 Navigation Functions $\ldots \ldots \ldots \ldots \ldots \ldots \ldots$

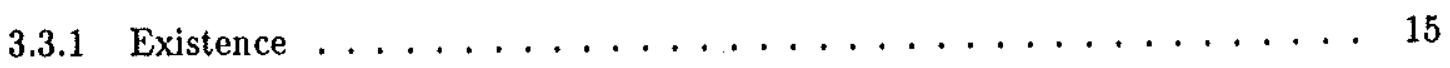




\subsubsection{Construction}

4 Applications of Total Energy as a Lyapunov Function

......... 17

411 The Mechanical Control System on $S O(3) \ldots \ldots \ldots \ldots$

4.1 .2 A Navigation Function on $S O(3) \ldots \ldots \ldots \ldots \ldots$

4.1 .3 Inverse Dynamics . . . . . . . . . . . . . . 21

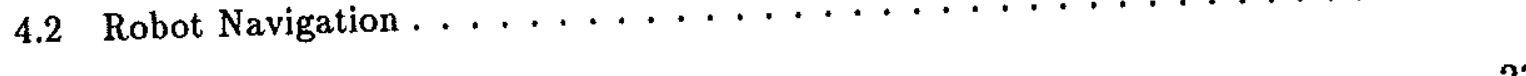

4.2.1 The Mechanical Control System ... . . . . . . . . . . 23

4.2.2 Navigation Functions on Euclidean Sphere Worlds . . . . . . . . . 24

4.2.3 Navigation Functions Induced by Diffeomorphism . . . . . . . . . . 25

5 Conclusion 


\section{Introduction}

It has been known for at least a century that the decrease in total energy of a dissipative mechanical system implies the local convergence of generalized position toward a minimum of the potential function. Lagrange demonstrated the stability of motion around the equilibrium state of a conservative system using total energy in 1788 [26]. Asymptotic stability of the potential field minima resulting from the introduction of dissipative forces to a conservative system was discussed by Lord Kelvin in 1886 [44]. These ideas were generalized by Lyapunov in his 1892 doctoral dissertation [28]. The use of total energy for control applications has been rediscovered many times in the engineering community since Lyapunov's ideas were first introduced by Kalman and Bertram [14]. For example, a two decade old paper by Pringle [36] paper concerns the applications of total energy to satellite control. Credit for first noting utility of artificial potential energy in robotics applications a decade ago would appear to be due Khatib [15]. Arimoto and colleagues [32, 42] contributed a precise demonstration that every minimum of the potential field is a local attractor of a dissipative mechanical system by application of LaSalle's Invariance Principle. Similar independent work of Van der Schaft [38] and this author [21] appeared subsequently. Of course, the familiar idea of energy dissipation and its consequences for local stability behavior is to be found in physics texts as well $[1,8]$.

In contrast, the extent to which global conclusions about the phase portrait of a mechanical system may be drawn from analysis of the total energy function appears not to have been addressed in the previous literature. Yet every mechanical system includes a lifted gradient vector field arising from its potential energy. Since the global limit set of a nondegenerate gradient vector field is trivial (consists of a finite number of isolated points) it seems reasonable to inquire whether such simple limit behavior lifts as well. This paper answers that question affirmatively and demonstrates the utility of the observation in the design of controllers for mechanical systems resulting in "almost global" asymptotically stable closed loops.

The mechanical systems form a large and important class of highly nonlinear finite dimensional dynamical plants which include, for example, all rigid link robots and satellites. Generally, the construction of feedback controllers for nonlinear systems which ensure that global convergence properties hold for the closed loop system is a hopeless task. Indeed, of the large and ever growing number of investigators who have exploited total energy as a Lyapunov function either for purposes of robotic manipulation $[32,12,16,35]$ or for satellite attitude control $[36,29,13]$, there seem to be none who systematically have considered the global aspects of the problem. Here, via elementary arguments from the theory of dynamical systems, we show that the limit properties of a dissipative mechanical system on the phase space reduce to the trivial case of gradient dynamics on the configuration space. Both, of course, are constrained to respect the configuration space topology, and the selection of useful classes of gradient systems requires some care. In the end, ensuring the "strongest" global convergence behavior allowed by the configuration space topology from a reasonably large set of initial phase space states is guaran teed by the construction of scalar cost functions endowed with certain "navigation properties."

The paper is organized as follows. The mechanical control system, $\Sigma$, is defined in Section 2 with some care paid to its attendant global geometric features. The dissipative mechanical 
system, $\Delta$, is shown to result from a state feedback law determined by a gradient system, $\Gamma$, along with a suitable dissipative vector field. The section ends with a recitation of Lord Kelvin's century old result (Theorem 1). Attention shifts to the global limit properties of the dissipative mechanical system, $\Delta$, in Section 3. The "navigation function" is introduced as a central tool of feedback controller design yielding a "congruence" between the limit sets of $\Delta$ and $\Gamma$ (Theorem 2). The section ends with the summary of our earlier result showing that smooth navigation functions always exist on smooth manifolds with boundary (Theorem 3). Application of these results to satellite attitude tracking, summarized by Theorem 4 , and to robot obstacle avoidance, summarized by Theorem 5 and Theorem 6 , is the concern of Section 4. 


\section{Dissipative Mechanical Systems}

The geometry of classical physics has been extensively studied for decades, and recent years have witnessed the publication of numerous expository texts containing the background material required for the present paper. This section reviews the relevant ideas, following very closely the presentation in the excellent text of Abraham and Marsden [1].

The setting for all the results presented in this paper is a configuration space, $\mathcal{J}$, a compact Riemannian manifold with boundary. Mechanical systems may be approached from the point of view of symplectic geometry on the cotangent bundle, $\sigma: T^{*} \mathcal{J} \rightarrow \mathcal{J}$, or Riemannian geometry on the tangent bundle, $\tau: T \mathcal{J} \rightarrow \mathcal{J}:$ it is the second point of view which will be most useful in this paper. A Lagrangian dynamical system is defined by the extrema of an integral cost functional applied to motion on the tangent bundle, $T \mathcal{J}$. Section 2.1 particularizes this idea to the instance of a mechanical system when the cost functional is defined in terms of a Riemannian metric, and there obtains the notion of kinetic energy. Adding a pointwise cost functional to the original configuration space introduces potential and total energy, discussed in Section 2.2. The dissipative vector fields are introduced in Section 2.3. Finally, the chief object of present study, the dissipative mechanical system, is defined in terms of all these ingredients.

\subsection{Mechanical Systems}

Given a Lagrangian function, $\lambda: T \mathcal{J} \rightarrow \mathbb{R}$, the Variational Principle of Hamilton [1], states that of all the curves, $b$, between two points, $q_{1}, q_{2} \in \mathcal{J}$, the one whose tangent curve, $c \triangleq T b$, minimizes

$$
\int \lambda \circ c
$$

also satisfies the classical Euler-Lagrange coordinate equations,

$$
\frac{d}{d t} D_{\dot{q}} \lambda-D_{q} \lambda=0
$$

These coordinate equations define a Lagrangian vector field on the tangent bundle,

$$
f_{\lambda}: T \mathcal{J} \rightarrow T T \mathcal{J}
$$

which constitutes a second order equation on $\mathcal{J}$ in the sense that $T \tau f=1_{T \mathcal{J}}$ [1].

\subsubsection{Kinetic Energy as a Riemannian Metric}

A particular Riemannian metric results from the choice of a morphism [10, Ch. 4.1], $M \in$ $\mathcal{M}\left[T \mathcal{J}, T^{*} \mathcal{J}\right]$, from the tangent bundle to the co-tangent bundle which is symmetric positive definite (i.e. $(M w, v)=(M v, w)$ and $(M v, v)>0$ for all $v, w \in T \mathcal{J}$ assuming that $\tau(v)=\tau(w)$ ). 
We now suppose that a distinguished symmetric positive definite morphism, $M$, has been chosen and denote the resulting inner product

$$
\langle v \mid w\rangle \triangleq(M v, w)
$$

This metric is understood to arise from the kinetic energy of the mechanical system, 1

$$
\kappa: T \mathcal{J} \rightarrow \mathbb{R} ; \quad v \mapsto \frac{1}{2}\langle v \mid v\rangle
$$

defined by the dynamical parameters of rigid bodies as determined by a kinematic relationship. For example, the author has presented a quick derivation of the Riemannian metric resulting from the sort of physical system which motivates the paper - the "kinematic chains" encountered in robotics - in a recent encyclopedia article [23]. Examples will be given in Section 4.

\subsubsection{The Mechanical Control System}

Define a mechanical system to be the Lagrangian dynamical system resulting from a cost functional $\lambda \triangleq \kappa$ specified by a kinetic energy - an analytic section of the tangent to the tangent bundle, $f_{\kappa}: T \mathcal{J} \rightarrow T T \mathcal{J}$. We now present a formal model expressing the manner in which control inputs may be applied to this system.

Suppose that the physical system is "actuated" by external forces which are under our control. In coordinates, the generalized forces, $u$, affect Lagrangian dynamics according to the relation

$$
\frac{d}{d t} D_{\dot{q}} \lambda-D_{q} \lambda=u
$$

The generalized forces may be modeled globally as points in the cotangent bundle, $\sigma: T^{*} \mathcal{J} \rightarrow \mathcal{J}$. However there is a formal problem in taking the space of combined inputs-states to be the simple cross product, $T^{*} \mathcal{J} \times T \mathcal{J}$, since a force, $u=\left(q_{1}, f\right) \in T_{q_{1}}^{*} \mathcal{J}$, cannot be applied at a phase, $v=\left(q_{2}, x\right) \in T_{q_{2}} \mathcal{J}$, unless both are vectors over the same configuration $-\sigma(u)=q_{1}=r(v)=$ $q s_{2} \in \mathcal{J} .^{2}$ To resolve this problem it seems most natural to adopt the point of view introduced by Brockett [3] and model the input-state space as the total space, $\mathcal{T} \triangleq \sigma^{*} T \mathcal{J}$, obtained by pulling back $T \mathcal{J}$ over $\sigma$, according to the following commutative diagram,

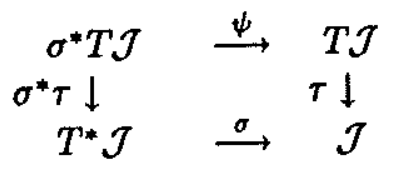

resulting in a vector bundle over the state space via the natural vector bundle map [10, Ch. 4.2] $\psi: \mathcal{T} \rightarrow T \mathcal{J}$. This simply means that $e \in \mathcal{T}$ is a valid input-state point if and only if its base,

\footnotetext{
${ }^{1}$ Kinetic Energy is formally defined as a scalar valued map on $T^{*} \mathcal{J}[1]$, however it will simplify the discussion and do no iechnical harm in the present paper to speak of $\kappa$ as a map on $T \mathcal{J}$.

${ }^{2}$ This problem may be resolved, of course, if $\mathcal{J}$ is parallelizable: the product notion will go through after the extra step of identifying $T \mathcal{J} \approx \mathcal{J} \times \mathbb{R}^{n}$ and $T^{*} \mathcal{J} \approx \mathcal{J} \times \mathbb{R}^{n}$ with the penalty of an extra coordinate transformation in all future computation.
} 
$u=\sigma^{*} \tau e$, and vector component, $v=\psi(e)$, are, in turn, vectors over the same configuration, $q=\sigma(u)=\tau(v)$.

Now, following Brockett, we are in a position to define the mechanical control system as a smooth section of the pullback of $T T \mathcal{J}$ through $\psi$,

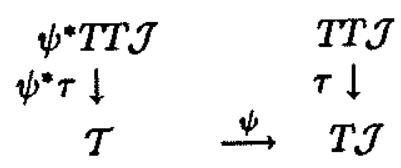

as follows. Equation (2) implies that the generalized forces, $u \in \mathcal{U}$, affect the Lagrangian vector field on $T T \mathcal{J}$ through vertical lift, $V_{v}: T_{\tau v} \mathcal{J} \rightarrow T_{v}(T \mathcal{J})[1$, Def. 3.7.5], of the vector field associated with $u \in T^{*} \mathcal{J}$ by the Riemannian metric, $M^{-1} u$. Thus, a mechanical control system, is the section $f_{\Sigma}: \mathcal{T} \rightarrow \psi^{*} T T \mathcal{J}$ determined by the ordered pair, consisting of a configuration space and a choice of kinetic energy,

$$
\Sigma \triangleq(\mathcal{J}, \kappa)
$$

defined by

$$
f_{\Sigma}\left(v_{u}\right) \triangleq f_{\kappa}(v)-V_{v} \circ M_{\tau v}^{-1} u
$$

Assuming, as we do throughout this paper, that $M$ is analytic, and that $\mathcal{J}$ is an analytic manifold, it follows that a mechanical control system (4) falls within the class of linear analytic control systems.

\subsection{Gradient Vector Fields in Mechanical Systems}

Consider the class of twice differentiable real valued functions $\varphi \in C^{2}[\mathcal{J}, \mathbb{R}]$ on a compact Riemannian manifold, $\mathcal{J}$. The co-vector field, $d \varphi$, is related to the gradient vector field, $\operatorname{grad} \varphi$, of $\varphi$ via the Riemannian metric inverse morphism,

$$
\operatorname{grad} \varphi \triangleq M^{-1} d \varphi \text {. }
$$

Thus, a gradient system is determined by the triple consisting of a configuration space, a Riemannian metric, and a scalar valued function,

$$
\Gamma \triangleq(\mathcal{J}, M, \varphi)
$$

yielding the vector field $\operatorname{grad} \varphi: \mathcal{J} \rightarrow T \mathcal{J}$

\subsubsection{Limit Behavior of Gradient Systems}

One calls $\varphi$ a Morse function if its hessian (matrix of second derivatives) is non-singular at every critical point [10]. Gradient vector fields resulting from Morse functions give rise to flows with simple limit behavior which may be summarized as follows. 
Proposition 2.1 Let $\varphi$ be a twice continuously differentiable Morse function on a compact Riemannian manifold, $\mathcal{J}$. Suppose that $\operatorname{grad} \varphi$ is transverse and directed away from the interior of $\mathcal{J}$ on any boundary of that set. Then the negative gradient flow has the following properties:

1. $\mathcal{J}$ is a positive invariant set;

2. the positive limit set of $\mathcal{J}$ consists of the critical points of $\varphi$;

3. there is a dense open set $\tilde{\mathcal{J}} \subset \mathcal{J}$ whose limit set consists of the local minima of $\varphi$,

Proof: $\quad$ Since the vector field is directed toward the interior of $\mathcal{J}$ on its boundaries by hypothesis, it follows that this set is positive invariant. The limit set for any trajectory of a gradient system is an equilibrium state [11], hence, in this case, a minimum, maximum, or saddle of $\varphi$ in the interior of $\mathcal{J}$. It remains to demonstrate the third property.

Since $\varphi$ is Morse, it has only isolated critical points and the number of local maxima and saddles is countable. Each of these has a stable manifold of lower dimension than $\mathcal{J}$, whose closure in $\mathcal{J}$ is consequently nowhere dense. But the domain of attraction of any maximum or saddle is contained in its stable manifold. It follows that the domain of attraction of the minima of $\varphi, \tilde{\mathcal{J}}$, is the complement of the countable union of nowhere dense sets in $\mathcal{J}$.

It is worth commenting on two technical assumptions in the hypothesis of this proposition. The first, the assumption of compactness, is a relatively harmless measure taken to guarantee that $\mathcal{J}$ is positive invariant with respect to the negative gradient flow. For consider the scalar function, $\varphi(q) \triangleq q^{3}$, defined on $\mathbb{R}$, yielding the the negative gradient dynamics,

$$
\dot{q}=-3 q^{2} \text {. }
$$

Since this system has finite escape trajectories, it is not true that $\mathbb{R}$ is positive invariant under the flow. However, there is no possibility of finite escape from a compact set without boundary (and the tranversality assumption precludes finite escape otherwise) as the proposition requires. In the case that the configuration space is homeomorphic to Euclidean n-space, a "radial unboundedness" condition on $\varphi$ [9] will yield equivalent results. However, for most mechanical systems of interest the configuration space (but, of course, not the phase space) is compact.

The more onerous requirement, that $\varphi$ be a Morse function, guarantees that the limit set of every motion is a single critical point. For, absent such a restriction, connected limit sets are known to be possible [34][Example 1.1.3]. Yet isolated critical points might still be degenerate: a degenerate saddle of $\varphi$ might well include an open set in its domain of attraction. Indeed, the previous example demonstrates the existence of a dynamical system whose unique isolated critical point is unstable, yet which has a domain of attraction which is open in the configuration space, $\mathbb{R}$. This possibility would invalidate the argument in the previous proof that any open set has a limit set comprised of minima. The Morse property guarantees that no co-dimension 
one set of saddle connections can separate $\mathcal{J}$. While this condition incurs an undesirable loss of generality ${ }^{3}$ the technical problems which result in its relaxation require more attention than worthwhile in this paper.

\subsubsection{Lifting Gradient Vector Fields}

Any smooth real valued function, $\varphi: \mathcal{J} \rightarrow \mathbb{R}$ may be "pulled back" to $T \mathcal{J}$ in a natural fashion by defining $\tilde{\varphi} \triangleq \varphi \circ \tau$. This defines a new Lagrangian function,

$$
\lambda \triangleq \kappa-\tilde{\varphi}
$$

whose Lagrangian vector field, $f_{\kappa-\tilde{\varphi}}$, can be shown to include a "lift" of the gradient vector field of $\varphi$

$$
f_{\kappa-\dot{\varphi}}(x)=f_{\kappa}(x)-V_{x}(\operatorname{grad} \varphi) \circ \tau(x),
$$

where $V$ is the vertical lift. It is in this sense that we are justified in claiming that mechanical systems provide a "natural analog computer" for gradient vector fields. In the absence of dissipative forces, this "integration" occurs, through the conservation of a total energy function,

$$
\eta \triangleq \kappa+\tilde{\varphi}
$$

in which sense $\varphi$ may be regarded as a potential energy function. ${ }^{4}$

Proposition 2.2 ( [1] ) The total energy, $\eta$ is invariant under the flow of the lagrangian vector field, that is,

$$
d \eta f_{\kappa-\bar{\varphi}} \equiv 0
$$

\subsection{Dissipative Mechanical Systems}

We now introduce the chief object of study in this paper, the dissipative mechanical system,

$$
\Delta \triangleq\left(\mathcal{J}, \eta, f_{d}\right)
$$

defined by the vector field, $f_{\Delta}: T \mathcal{J} \rightarrow T T \mathcal{J}$,

$$
f_{\Delta} \triangleq f_{\kappa-\bar{\varphi}}+f_{d}
$$

where $f_{\kappa-\tilde{\varphi}}$ is the Lagrangian vector field introduced in Section 2.2.2 and $f_{d}$ is a dissipative vector field to be defined below. The aim here is to show how $\Delta$ arises from the closed loop of a certain class of feedback controllers for $\Sigma$.

\footnotetext{
${ }^{3}$ Redundant degrees of freedom in robotics will result in non-immersive output (kinematic) maps. Thus, the pullback of a nondegenerate "task specification" vector field in the output space, $\mathbb{R}^{3} \times S O(3)$ will necessarily have degenerate equilibrium states in the configuration space.

"It is interesting to note that the base integral curves of $f_{\kappa-\varphi}$ are the geodesics of the Jacobi metric, $M_{\varphi} \triangleq\left(\varphi_{0}-\varphi\right) M$, where $M$ is the original metric, and $\varphi_{0}$ is any real value outside the range of $\varphi$ (possibly restricted to a suitably defined compact positive invariant submanifold of $\mathcal{J}$ ) [1][Thm. 3.7.7]. However, this point of view does not seem to lead to any new understanding in the present situation.
} 


\subsubsection{Dissipative Vector Fields and Feedback}

The last ingredient we require is the notion of a dissipative vector field: a vector field on the phase space, $f_{d}: T \mathcal{J} \rightarrow T T \mathcal{J}$, which is "vertical" (i.e. $T \tau f_{d}=0$ ), and acts negatively on kinetic energy, $d \kappa f_{d} \leq 0[1]$. Since $f_{d}$ is vertical it follows that there exists a morphism, $G_{d} \in \mathcal{M}^{\infty}\left[T \mathcal{J}, T^{*} \mathcal{J}\right]$, such that

$$
f_{d}(v)=V_{v} \circ M_{\tau v}^{-1} \circ G_{d}(v)
$$

Thus, considering the following analytic section of the total space $\psi: \mathcal{T} \rightarrow T \mathcal{J}$,

$$
g(v) \triangleq G_{d}(v)+d \varphi \circ r(v),
$$

as a feedback law relating states to inputs, it is clear that the dissipative mechanical system on the state space, $f_{\Delta}$, is the closed loop system resulting from the composition

$$
f_{\Delta}=f_{\Sigma} \circ g
$$

In coordinates, $p: T \mathcal{J} \rightarrow \mathcal{O}$, for some convex open subset of $\mathbb{R}^{n}, \Delta$ gives rise to the differential equation

$$
\begin{aligned}
& \dot{p_{1}}=p_{2} \\
& \dot{p_{2}}=-\underline{M}^{-1}\left(p_{1}\right)\left(\left[C\left(p_{1}, p_{2}\right)+\underline{K}_{2}\left(p_{1}, p_{2}\right)\right] p_{2}+\underline{K}_{1}\left(p_{1}\right) p_{1}\right)
\end{aligned}
$$

where $C$ denotes the coriolis forces, $\underline{K}_{2}$ the damping law, and $\underline{K}_{1}$ the spring law.

\subsubsection{Local Limit Behavior}

In the presence of dissipative forces, integration of dissipative mechanical system, $\Delta$, produces local limit behavior in the phase space, $T \mathcal{J}$ which is analogous to that of the original gradient system, $\Gamma$, in the configuration space, $\mathcal{J}$. This notion is made precise by the following familiar result.

Theorem 1 ( Lord Kelvin (1886) [44, §345]) If $q_{0}$ is a local minimum of $\varphi$ in $\mathcal{J}$, then $\left(q_{0}, 0\right)$ is a stable equilibrium state of the dissipative mechanical system, $\Delta(9)$, in $T \mathcal{J}$. In particular, $\eta(8)$ is a Lyapunov function for $f_{\Delta}$, with with Lie derivative

$$
L_{f_{\Delta}}(\eta)=d \kappa f_{d}
$$

Since $\eta$ is not a strict Lyapunov function - that is, $d \kappa f_{d}$ must vanish on the entire zero section of $T \mathcal{J}$ and the Lie derivative, $L_{f}(\eta)$ is negative semi-definite - rigorous conclusions about asymptotic properties of the flow near $\left(q_{0}, 0\right)$ require the application of LaSalle's Invariance Principle [27] as shown, for example, by Arimoto and colleagues [42]. 


\section{Global Limit Properties of Dissipative Mechanical Systems}

The global phase portrait of a regular gradient system, $\Gamma(5)$, is as simple as one could imagine arising from a nonlinear differential equation. The feedback synthesis procedure introduced (11) for the class of mechanical control systems, $\Sigma(3)$, results in a closed loop dissipative mechanical system, $\Delta(9)$, containing a "lift" of a constituent gradient vector field, grad $\varphi$. This Section will demonstrate that an appropriately chosen potential function, $\varphi$, called a "navigation function," induces limit behavior of $\Delta$ on a useful subset of the phase space, $T \mathcal{J}$, which may be identified with that of $\Gamma$ on the configuration space, $\mathcal{J}$. Thus, at least with regard to steady state behavior, our synthesis procedure reduces the control designer's task from the consideration of complex hamiltonian dynamics to the manipulation of comparatively trivial gradient dynamics.

This Section is organized roughly congruently to Proposition 2.1 which we take as our model of desired steady state behavior. We first show that $f_{\Delta}$ fails to be transverse on the boundary of phase space, and find a reasonable substitute for this condition in Section 3.1. We next show in Section 3.2 that the global limit set of $\Delta$ is a lift of that of $\Gamma$ into the phase space. This yields a global version of Lord Kelvin's result in the form of Proposition 3.6. We finally introduce the notion of a navigation function in Section 3.3. This restores to $\Delta$ a positive invariant set which includes all of the (lifted) configuration space and yields convergence properties which are as strong as the topology of $\mathcal{J}$ allows.

\subsection{Positive Invariance}

To begin with, we must preclude the possibility of finite escape: the transversality of grad $\varphi$ on $\partial \mathcal{J}$ is no longer enough. Intuitively, it is helpful to think of a marble rolling along a hilly terrain in the earth's gravitational field through a viscous atmosphere. Tranversality simply implies that the terrain slopes away from any forbidden region (the boundary of configuration space): a marble traveling with sufficient kinetic energy could, of course, roll uphill and crash through the boundary. We first give an example, and then establish a reasonably large subset of the phase space which is positive invariant under the flow induced by $\Delta$.

\subsubsection{Rolling Uphill}

As an example, take $\mathcal{J}$ to be the closed real interval, $[-\epsilon, 1]$, with the Euclidean metric as kinetic energy, $\kappa \triangleq \frac{1}{2} \dot{q}^{2}$, and define the potential energy to be $\varphi \triangleq \frac{1}{2} q^{2}$. The gradient vector field, $\operatorname{grad} \varphi=q$, is transverse and exterior directed on $\partial \mathcal{J}=\{-\epsilon, 1\}$ as required by Proposition 2.1 as long as $\epsilon>0$. Now, using the coordinate system,

$$
p=\left[p_{1}, p_{2}\right]^{\mathrm{T}} \in T \mathcal{J}=[-\epsilon, 1] \times \mathbb{R},
$$

choose a damping law $G(p) \triangleq \delta p_{2}$ corresponding to a valid dissipative vector field, $f_{d}(v)=$ $\left[0,-\delta v_{2}\right]^{\mathrm{T}}$, as long as $\delta>0$. 
The associated dissipative mechanical system, $\Delta$, is expressed in coordinates as as

$$
\dot{p}=f_{\Delta}(p)=\left[\begin{array}{cc}
0 & 1 \\
-1 & -\delta
\end{array}\right] p
$$

Now the boundary of the phase space is the union of the two parallel lines

$$
\partial(T \mathcal{J})=T_{\partial \mathcal{J}} \mathcal{J}=(\{-\epsilon\} \times \mathbb{R}) \cup(\{1\} \times \mathbb{R}),
$$

on which $f_{\Delta}$ fails to be transverse at the points $[-\epsilon, 0],[1,0]$. In fact, $f_{\Delta}$ is directed away from the interior of the phase space - an unfortunate circumstance - on the upper half of the line through $[1,0]$ and the lower half of the line through $[-\epsilon, 0]$. Consequently, it may be observed that the trajectory of $f_{\Delta}$ through every initial condition in a neighborhood of these open half line segments must escape from $T \mathcal{J}$ in finite time. Thus, the transversality of the gradient field on $\partial \mathcal{J}$ fails to guarantee that a dissipative mechanical system gives rise to a complete dynamical system on $T \mathcal{J}$. This example holds true in general, for exactly the same simple reason, as the following result demonstrates.

Lemma 3.1 If $\partial \mathcal{J} \neq \emptyset$ then $T \mathcal{J}$ contains an open set of points whose trajectories under $\Delta$ exhibit finite escape.

Proof: It will suffice to show that there is a regular point of $f_{\Delta}$ in $\partial(T \mathcal{J})$ whose forward trajectory leaves $T \mathcal{J}$ in finite time - the open set is then contained within the local flow box $[11, \mathrm{Ch} .11 .2]$ around that point.

No point away from the zero section of $T \mathcal{J}$ can be an equilibrium state of $f_{\Delta}$, so consider the curve $c_{+}(t)$, the forward trajectory of $f_{\Delta}$ through $v \in \partial(T \mathcal{J})$. We may assume with no loss of generality that $v$ is not tangent to $\partial \mathcal{J}-$ that is $v \in T_{\tau v} \mathcal{J}-T_{\tau v} \partial \mathcal{J}$. Its base curve, $b_{+}(t) \triangleq \tau c(t)$, has the property that $b_{+}(0) \in \partial \mathcal{J}$. We have $\dot{b}_{+}(0)=T \tau f_{\Delta}(v)=v$ since $f_{\Delta}$ is second order as shown in Section 2. On the other hand, if $b_{-}(t)$ is the base curve of the forward trajectory through $-v$ then $\dot{b}_{-}(0)=-v$ in exactly the same way. Thus, either the curve $b_{-}$or $b_{+}$leaves $\mathcal{J}$ after $t=0$ from which it follows that either $c_{-}$ or $c_{+}$leaves $T \mathcal{J}$ after $t=0$.

\subsubsection{Invariance of the Lowest Boundary Energy Set}

It is clear that $f_{\Delta}$ makes the boundary of $T \mathcal{J}$ "dangerous" away from the zero section. We now provide a simple means of characterizing positive invariant subsets of the phase space which intersect its boundary at most at points in the zero section. 
Proposition 3.2 Let $\varphi \in C^{2}[\mathcal{J}, \mathbb{R}]$ and suppose that

$$
b_{1} \triangleq \inf _{q \in \partial \mathcal{J}} \varphi(q)
$$

is a regular value of $\varphi$ (and is understood to be $+\infty$ if $\partial \mathcal{J}=\emptyset$ ) and that

$$
b_{1}>b_{0} \triangleq \inf _{q \in \mathcal{J}} \varphi(q) .
$$

Then for all constants, $b \in\left(b_{0}, b_{1}\right]$, the set of "bounded total energy" states of $\eta(8)$,

$$
\mathcal{E}^{b} \triangleq\{v \in T \mathcal{J}: \eta(v) \leq b\}
$$

is a positive invariant set of the dissipative mechanical system (9), with non-empty interior.

Proof: $\quad$ Choosing $b>b_{0}$ guarantees that $\mathcal{E}^{b}$ has a non-empty interior. For $\eta^{-1}\left(b_{0}, b\right) \subseteq \mathcal{E}^{b}$ is both non-empty ( it contains the set identified with $\emptyset \neq \varphi^{-1}\left(b_{0}, b\right)$ in the zero section) and open ( $\eta$ is continuous).

To demonstrate the positive invariant property, it suffices to show that $\partial \mathcal{E}^{b} \subseteq \eta^{-1}[c]$, for $d \eta f_{\Delta} \leq 0$ according to Theorem 1 . To see that the boundary ${ }^{5}$ is contained in the level set, as claimed, examine its partition,

$$
\partial \mathcal{E}^{b}=\left(\partial(T \mathcal{J}) \cap \partial \mathcal{E}^{b}\right) \cup\left(\dot{T}^{\circ} \mathcal{J} \cap \partial \mathcal{E}^{b}\right)
$$

The first component is equivalent to $\partial(T \mathcal{J}) \cap \mathcal{E}^{b}$ which, in turn, is equivalent to $T_{\partial \mathcal{J}} \mathcal{J} \cap \mathcal{E}^{b}$. Now this set is entirely contained in the subset of the zero section of $T \mathcal{J}$ identified with $\varphi^{-1}[b] \cap \partial \mathcal{J}$. For $v \in T_{\partial \mathcal{J}} \mathcal{J}$ implies $\varphi \circ \tau(v) \geq b_{1} \geq b$ since $b_{1}$ is the infimal value of $\varphi$ on $\mathcal{J}$ by hypothesis. But $\eta(v)>\varphi \circ \tau(v)$ unless $v \in \kappa^{-1}[0]$, in which case $\eta(v)=\varphi \circ \tau(v)$ and we have $\tau(v) \in \varphi^{-1}[b]$. This demonstrates that the first component is equivalent to $\partial T \mathcal{J} \cap \eta^{-1}[b]$ (and is empty when $b<b_{1}$ ).

It remains to show that $\stackrel{\circ}{T \mathcal{J}} \cap \partial \mathcal{E}^{b} \subseteq T^{\circ} \mathcal{J} \cap \eta^{-1}[b]$ : specifically, notice that any point not in the second set cannot be in the first. This is so, for if $v \in T \mathcal{J} \cap\left(\mathcal{E}^{b}-\eta^{-1}[b]\right)$ then it is necessarily in the inverse image of some open interval, $v \in \eta^{-1}[\eta(v)-\epsilon, \eta(v)+\epsilon]$, which is an open set in $T^{\circ} \mathcal{J} \cap \mathcal{E}^{b}$ since $\eta$ is continuous.

With respect to the example of Section 3.1.1, note that $b_{1}=\frac{1}{2} \epsilon^{2}$, and $b_{0}=0$, so that the positive invariant sets, $\mathcal{E}^{b}$ defined by the proposition are simply the closed disks around the origin of $\mathbb{R}^{2}$ whose radius is less than or equal to $\epsilon$.

\footnotetext{
${ }^{3}$ Strictly speaking, the boundary of $\mathcal{E}^{b}$, considered as a point set, has no intersection with the boundary of the manifold in which it lies, $T \mathcal{J}$. To avoid the technicalities involved in distinguishing the two different notions of boundary, we assume that the original space $T \mathcal{J}$ is collared [10], thus both $T \mathcal{J}$ and $\mathcal{E}^{b}$ are closed subsets within the open collared set, $\widetilde{T J}$ and the boundary operations are taken in the sense of point set topology so that the two subsets do indeed share a common boundary component as described.
} 


\subsection{Limit Behavior}

The hypothesis of Proposition 3.2 in some sense strengthens that required for conclusion (1) of Proposition 2.1: if $b_{1}$ is a regular value and a minimal value on the boundary, then grad $\varphi$ must be transverse to $\partial \mathcal{J}$ at points near $\varphi^{-1}\left(b_{1}\right)$. In contrast, once sufficient conditions for positive invariance have established, a conclusion analogous to (2) for Proposition 2.1 follows quite easily: the critical points constitute the entire positive limit set, and their local stability properties are inherited directly from that of $\varphi$.

\subsubsection{The Limit Set Consists of Equilibrium States}

In general, Hamiltonian systems will have extremely complicated limit behavior. The addition of the damping term, $f_{d}$, makes the global limit behavior of the dissipative mechanical system $\Delta$ as trivial as that of its gradient model, $\Gamma$. The formal argument below is adapted from the discussion of gradient vector fields in [34, Example 1.1.3]. Somewhat surprisingly, the reasoning there applies to the present situation almost without change.

Proposition 3.3 The positive limit set of any positive invariant subset of $T \mathcal{J}$ under the flow of a dissipative mechanical system, $\Delta$, consists of the critical points of $\varphi$ identified with their image in the zero section of $T \mathcal{J}$.

Proof: It is clear that the equilibrium states of $f_{\Delta}$ correspond exactly to the critical points of $\varphi$, hence, form a totally isolated set in the zero section of $T \mathcal{J}$. Now suppose some initial condition, $v_{0}$, has a limit set which includes a state, $v_{1}$, whereon $f_{\Delta}$ does not vanish. It is clear that $v_{1}$ must be a regular point of $\eta$ since the critical points of the latter correspond exactly to the critical points of $\varphi$ (lifted into the zero section). Therefore, according to the implicit function theorem, the intersection of $\eta^{-1}\left[\eta\left(v_{1}\right)\right]$ with a sufficiently small neighborhood of $v_{1}$ is a codimension 1 submanifold of $T \mathcal{J}$. The computation of $\dot{\eta}$ in Theorem 1 demonstrates that the flow of $f_{\Delta}$ is transverse to this submanifold in a small enough neighborhood of $v_{1}$. Yet, since that point is in the limit set of $v_{0}$, the trajectory through $v_{0}$ must intersect this submanifold in more than one point (in fact, an infinity of points). This would contradict the monotone property of $\eta$ on the flow.

\subsubsection{Local Stability of the Equilibrium States}

Lord Kelvin's result, Theorem 1, demonstrates that the minima of $\varphi$ (suitably identified with points in the zero section of $T \mathcal{J}$ ) are local attractors. To gain a conclusion analogous to (3) in Proposition 2.1 it must be verified as well that local behavior of the negative gradient flow near 
a critical point other than a minimum is a model for local behavior of the dissipative mechanical system near the identified equilibrium state. To see this, first observe that the local linearized vector field at any equilibrium state of the dissipative mechanical system, $\Delta(9)$, is the linear time invariant dissipative mechanical system determined by the hessian of the potential function at that point.

Lemma 3.4 Let $q_{0}$ be a critical point of $\varphi$. Then the linearized vector field at the equilibrium state, $\left(q_{0}, 0\right)$, of the dissipative mechanical field, (12), is given in local coordinates as

$$
\left[D f_{\Delta}\right]\left(q_{0}, 0\right)=\left[\begin{array}{cc}
0 & I \\
-\underline{M}\left(q_{0}\right)^{-1} \underline{K}_{1}\left(q_{0}\right) & -\underline{M}\left(q_{0}\right)^{-1} \underline{K}_{2}\left(q_{0}, 0\right)
\end{array}\right],
$$

where $\underline{K}_{1}=D^{2} \varphi$ is the hessian matrix of $\varphi, \underline{K}_{2}$ is the local matrix representation of the linearized dissipative field, and $\underline{M}$ is the matrix representation of the kinetic energy metric, as introduced in (10).

If $M$ is a metric, then $\underline{M}\left(q_{0}\right)$ is a positive definite symmetric matrix. Similarly, if $f_{d}$ is strict, then $K_{2}(0,0)$ has a positive definite symmetric part by assumption. Thus the local stability properties of equilibria of $f$ are determined by the nature of the Hessian at the identified critical point of $\varphi$ as the following result indicates: that is, the stability of the origin of a linear time invariant dissipative mechanical system in $\mathbb{R}^{2 n}$ is governed by the stability properties of the associated gradient system in $\mathbb{R}^{n}$.

Lemma 3.5 ( Chetaev [4] ) Let $M, K_{2}, K_{1}$ be symmetric matrices in $\mathbb{R}^{n \times n}$ and let $M, K_{2}$ be positive definite. The origin of the linear time invariant system in $\mathbb{R}^{2 n}$,

$$
\left[\begin{array}{c}
\dot{x_{1}} \\
\dot{x_{1}}
\end{array}\right]=\left[\begin{array}{cc}
0 & I \\
-M^{-1} K_{1} & -M^{-1} K_{2}
\end{array}\right]\left[\begin{array}{l}
x_{1} \\
x_{2}
\end{array}\right]
$$

has one of the following stability properties: (i) asymptotically stable; (ii) stable but not attractive; (iii) unstable; if and only if the origin of the linear time invariant system in $\mathbb{R}^{n}$,

$$
\dot{x_{1}}=-K_{1} x_{1} \text {. }
$$

has the corresponding property.

Putting these results together yields a global version of Lord Kelvin's result which compares favorably in strength to Proposition 2.1: the significant difference is in the stronger boundary condition required by the present version as motivated by the discusion surrounding Proposition 3.2 .

Proposition 3.6 Let $\Delta$ be a dissipative mechanical system (9). Suppose that

$$
b_{1} \triangleq \inf _{q \in \partial \mathcal{J}} \varphi(q),
$$


is a regular value of $\varphi$ (and is understood to be infinite if $\partial \mathcal{J}=\emptyset$ ) and that

$$
b_{1}>b_{0} \triangleq \inf _{q \in \mathcal{J}} \varphi(q) .
$$

Then for all constants, $c \in\left(b_{0}, b_{1}\right]$, the set of "bounded total energy" states of $\eta(8)$,

$$
\mathcal{E}^{b} \triangleq\{v \in T \mathcal{J}: \eta \leq c\}
$$

has the following properties with respect to the flow of $\Delta$ :

1. $\mathcal{E}^{b}$ is a positive invariant set with non-empty interior;

2. the positive limit set of $\mathcal{E}^{b}$ consists of those points in the zero section of $T \mathcal{J} \cap \mathcal{E}^{b}$ identified with a critical point of $\varphi$;

9. there is a dense open set in $\mathcal{E}^{b}$ whose limit set consists of those points in the zero section of $T \mathcal{J} \cap \mathcal{E}^{b}$ identified with a local minimum of $\varphi$;

\subsection{Navigation Functions}

Proposition 3.6 suggests that the major effort in the construction of feedback controllers for mechanical systems will be spent finding suitable scalar valued functions, $\varphi$. In this section we address the following loosely posed control problem. Given a mechanical system, $\Sigma=(\mathcal{J}, \kappa)$ and a desired configuration, $q_{d} \in \mathcal{J}$ (identified, as usual, as a point in the zero section of $T \mathcal{J}$ ), to which we want to bring the system, what is a suitable choice of $\varphi$ ?

From the point of view of dynamical systems theory, linear feedback controllers for linear time invariant plants provide the means by which a desired equilibrium state of the closed loop system is made to attract the entire state space. The preceding results suggest two important relative deficiencies in the case of the mechanical control systems, $\Sigma$. First, no smooth vector field can have a global attractor unless the state space on which it is defined is homeomorphic to $\mathbb{R}^{n}[2]$. Thus, as is generally the case in robotics and other applications of mechanical systems theory, when the configuration space, $\mathcal{J}$, (and, hence, the state space, $T \mathcal{J}$ ) is not a homeomorph of a Euclidean vector space, there can be no hope of global asymptotic stability. Second, when the configuration space has a boundary, Lemma 3.1 demonstrates that there does not even seem to be the possibility of avoiding finite escape from the entire phase space (without recourse to unbounded vector fields which we must discard as unrealizable).

We now offer a definition placing conditions on $\varphi$ that result in what is arguably the "best possible" convergence behavior: we will ensure that the enirety of $\mathcal{J}$ (identified, as usual, with the zero section of $T \mathcal{J}$ ) is contained in a positive invariant subset of the phase space and will guarantee "almost global" asymptotic stability within that set.

Following Hirsch [10, Ch. 6.3], say that a smooth Morse function from a compact manifold, $\mathcal{F}$, is admissible over the interval $[a, b]$ if $\partial \mathcal{F}=\varphi^{-1}[a] \cup \varphi^{-1}[b]$, and $a, b$ are regular values. 
This implies that each of $\varphi^{-1}[a], \varphi^{-1}[b]$ is a union of components of $\partial \mathcal{F}$, a regular set on which $\operatorname{grad} \varphi$ is transverse. Note that if either level set is empty then the (corresponding) minimum or maximum value of $\varphi$ is taken only at critical points in the interior of $\mathcal{F}$. Adapting the terminology of Morse [33], say that $\varphi$ is polar if it has a unique minimum.

Definition 1 Let $\mathcal{F} \subset \mathrm{E}^{n}$ be a smooth compact connected manifold with boundary, and $q_{d} \in \dot{\mathcal{F}}$, be a distinguished point in its interior. A polar Morse function, $\varphi \in C^{2}[\mathcal{F},[0,1]]$, which is admissible over the interval $[-\epsilon, 1], \in \in \mathbb{R}^{+}$, and takes its unique minimum at $\varphi\left(q_{d}\right)=0$, is called a navigation function.

As a direct consequence of Proposition 3.6 we have the following result.

Theorem 2 Let $\Delta=\left(\mathcal{J}, \eta, f_{d}\right)$ be a dissipative mechanical system (9), and suppose that $\varphi$ is a navigation function for $\mathcal{J}$. Then the entire zero section of $T \mathcal{J}$ is included in the closed subset, $\eta^{-1}[0,1] \subseteq T \mathcal{J}$, which is positive invariant with respect to the flow induced by $\Delta$ (and includes the entirety of $T \mathcal{J}$ if $\mathcal{J}$ has no boundary). Moreover, there is an open dense set in $\eta^{-1}[0,1]$ whose limit set is exactly the desired configuration, $q_{d}$, at zero velocity.

\subsubsection{Existence}

When does a compact manifold with boundary admit a navigation function? In a recent paper [25] we have been able to answer this question quite unequivocally: we show that smooth navigation functions exist on any such smooth manifold for any desired interior point, $q_{d}$. We now review this result.

Smale proved the generalized "Poincaré's Conjecture" in higher dimensions roughly three decades ago. In so doing, he was led to develop a number of results concerning gradient systems of which the most important to us is the existence of "nice" functions. Suppose $\mathcal{M}$ is a smooth compact $n$-dimensional manifold whose boundary is the disjoint union of two closed components, $\partial \mathcal{M}=\mathcal{V}_{1} \cup \mathcal{V}_{2}$. Smale calls a smooth Morse function, $\varphi \in C^{\infty}[\mathcal{M}, \mathbb{R}]$, nice if $\varphi\left(\mathcal{V}_{1}\right)=-\frac{1}{2}$, $\varphi\left(\mathcal{V}_{2}\right)=n+\frac{1}{2}$, and at a critical point $p$ of $\varphi, \varphi(p)=$ index $p$. He obtains a number of important results with this construction, including a generalization of the somewhat earlier result of Morse which demonstrates that every smooth manifold with no boundary admits a smooth polar nondegenerate function [33]. For our purposes, this result is important if it can be extended to the general case with boundary.

The desired extension obtains by applying the notion of "cancellation" of adjacent (in index) critical points that Morse and Smale developed in the course of their independent investigations. A (reasonably) self-contained exegesis upon these techniques is provided by Milnor [30], whose version may be rendered as follows. Suppose that $\varphi$ is a smooth Morse function on $\mathcal{M}$ with two distinct interior critical points, $p_{1}$ and $p_{2}$, with indices $\lambda_{1}, \lambda_{2}$, respectively, possessing the 
properties $\lambda_{1} \neq \lambda_{2}$ and $\varphi\left(p_{1}\right) \neq \varphi\left(p_{2}\right)$. These two points may be cancelled if there exists another smooth Morse function, $\varphi^{\prime}$, on $\mathcal{M}$, which agrees with $\varphi$ everywhere away from a neighborhood of $\varphi^{-1}\left[\varphi\left(p_{1}\right)\right]$ and $\varphi^{-1}\left[\varphi\left(p_{2}\right)\right]$ in $\mathcal{M}$, yet which has two fewer critical points - one less critical point of index $\lambda_{1}$; one less critical point of index $\lambda_{2}$. It turns out that pairs of index 0 and index 1 critical points may be cancelled if the "lower boundary" has the right homology type [30, Thm. 8.1]. Moreover, there are always "enough" index 1 critical points to cancel all the minima if the manifold is connected: a proof may be found in [25] and was suggested to us by W. Massey. The application of these results in the present setting was suggested by M. Hirsch.

Theorem 3 ([25]) For every smooth compact connected manifold with boundary, $\mathcal{M}$, and any

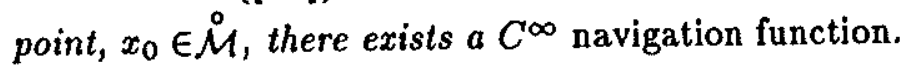

\subsubsection{Construction}

Although questions of existence presumably have independent mathematical interest, the engineer is only concerned to know of a negative outcome: in the present case, since we are guaranteed that what we seek is available, attention shifts to the question of construction. Here, we are led to impose some additional constraints.

We have already observed in Section 2.1.2 that the mechanical control system, $\Sigma(3)$, falls within the class of linear analytic systems. Since $\Delta$ results from the application of feedback, $g$ (11), to $f_{\Sigma}$, as described in Section 2.3 , it seemts only natural to demand that $g$ be analytic as well. This, of course, implies that $\varphi$ be not merely smooth, but analytic as well. Moreover, in their pioneering solution to the geometric robot navigation problem, Schwartz and Sharir [39] have argued persuasively that the class of real algebraic functions on real semi-algebraic varieties provides a practicable notion of "effective computation." We will adopt this point of view and insist that our constructions remain within the class of real algebraic functions: that is to say, the level set of a navigation function must be real semi-algebraic. Thus, the standard appeal to smooth functions "patched together" through a partition of unity will not avail. We are confronted, instead, with the harder task of building analytic algebraic navigation functions. 


\section{Applications of Total Energy as a Lyapunov Function}

This section presents two engineering applications of the preceding results. In both cases the configuration space fails to be a homeomorph of $\mathbb{R}^{n}$, so that global asymptotic stability is impossible: we display analytic algebraic navigation functions resulting in closed loop dynamics which are dissipative mechanical systems whose limit behavior accomplishes the specified task. In the first case, the problem of satellite attitude tracking discussed in Section 4.1 (and taken from the longer treatment of [18]), the configuration space is a Lie group - the entirety of $S O(3)$. Since the boundary is empty, convergence is guaranteed from almost every initial phase. To the best of the author's knowledge, Theorem 4 represents the first feedback controller for a fully actuated satellite which is well defined on the entirety of $S O(3)$ and which achieves asymptotically exact attitude tracking around an arbitrary reference trajectory with probability one. In the second case, the problem of robot obstacle avoidance discussed in Section 4.2 (and taken from our ealier publications $[25,37]$ ), the configuration space is a a subset of $\mathbb{R}^{n}$ (a Lie group as well) but this time it is bounded by a finite number of disjoint spheres. Here, the construction of navigation functions results in a robot which is guaranteed to approach a desired destination point in a cluttered space without hitting any of the clutter from every zero velocity initial condition excepting a set of zero measure. Again, to the best of the author's knowledge, Theorem 6 represents the first feedback controller which solves the global robot obstacle avoidance problem on nontrivial spaces of arbitary dimension.

\subsection{Satellite Attitude Tracking}

Now consider the application of the previous results to a classical control problem - asymptotically exact tracking - in a non-classical setting - the group of spatial rotations, $S O(3)$. Suppose there is a single rigid body actuated by three independent gas jets operating outside of the earth's gravitational field: the only forces operating on the body are the controlled inputs from the actuators which are capable of delivering any desired force in the "wrench space" of the body, TSO(3). Both the position and the velocity of the body are available from sensors. It is desired to force the body to track an arbitrary but entirely known reference trajectory. Since the system is completely actuated, there is perfect state information, and all derivatives of the reference trajectory are known, the velocity tracking problem is trivial. Namely, all nonlinearities due to the kinetic energy may be exactly cancelled, leaving a completely decoupled linear time invariant system. This procedure may be recognized as a trivial implementation of the global exact linearization techniques which have become so popular in the nonlinear control literature.

Consider, instead, the problem of attitude tracking. Namely, given a desired motion, $D \in$ $C^{2}[\mathbb{R}, S O(3)]$, construct a time invariant controller which causes the actual attitude to asymptotically approach $D(t)$ from any initial configuration, $A \in S O(3)$. So different is this from the trivial linear problem to which velocity tracking reduces that it is unsolvable as posed in that context. For, consider the particular case that $D(t)=D^{*}$ is some constant point. We seek a controller which makes that point (at zero angular velocity) a global attractor of the closed 
loop dynamics. Now, as was mentioned above, the domain of attraction of an attracting point is homeomorphic to some Euclidean vector space [2]. But the state space of our mechanical system - the tangent bundle over the rotation group - is clearly not homeomorphic to any Euclidean vector space. Thus, it would be impossible for our closed loop system to bring all initial conditions to the desired attitude. Evidently, the control system arising from a single rigid body is not globally linearizable by any technique since its state space is not a vector space. Our problem statement must be refined.

Since $S O(3)$ is a compact odd dimensional manifold without boundary, its Euler characteristic is zero [10]. It follows from the Theorem of Hopf [31] that any nondegenerate vector field on $S O(3)$ with an attracting equilibrium state has at least one other singularity which, if it is the only additional equilibrium state, must be totally unstable. Excepting the complement of some open dense set - in this case, the repelling point - trajectories of such a vector field are guaranteed to asymptotically approach the attracting point. Thus, although topological obstructions preclude a globally asymptotically stable system, a practically equivalent formulation which respects the underlying topology of the problem may be attainable. Say that a dynamical system is almost globally asymptotically stable if all trajectories starting in some open dense subset of the state space tend asymptotically to a specified stable equilibrium state. This we take as the criterion of convergence for our tracking algorithms on $S O(3)$ : it is the best possible result. Moreover, Theorem 2 assures us that we may achieve this result by recourse to feedback of the form (11) if we find a navigation function for $S O(3)$. That is the task we now undertake.

\subsubsection{The Mechanical Control System on $S O(3)$}

The configuration space of a rigid body is the group of rigid transformations, $S O(3) \times \mathbb{R}^{3}$. If we are concerned only with the attitude of a rigid body, then it suffices to treat $S O(3)$ alone, which we now identitfy with a subset of $\mathbb{R}^{9}$,

$$
S O(3) \triangleq\left\{R \in \mathbb{R}^{3 \times 3}: R^{\mathrm{T}} R=I \text { and }|R|=1\right\} .
$$

This Lie group has as its Lie algebra the set of skew symmetric matrices,

$$
\operatorname{so}(3)=\operatorname{skew}(3) \triangleq\left\{J \in \mathbb{R}^{3 \times 3}: J+J^{\mathrm{T}}=0\right\},
$$

which is isomorphic to $\mathbb{R}^{3}$ according to the linear bijection

$$
J:\left[\begin{array}{l}
w_{1} \\
w_{2} \\
w_{3}
\end{array}\right] \mapsto\left[\begin{array}{ccc}
0 & -w_{3} & w_{2} \\
w_{3} & 0 & -w_{1} \\
-w_{2} & w_{1} & 0
\end{array}\right]
$$

The vector space of three by three matrices is the direct sum,

$$
\mathrm{R}^{3 \times 3}=\operatorname{sym}(3) \oplus \operatorname{skew}(3)
$$


of the symmetric and skew-symmetric matrices. Thus, we may define a unique "pseudo-inverse" for $J$ whose domain is extended to all of $\mathbb{R}^{3 \times 3}$ by projection onto the linear subspace, skew (3).

$$
J^{\dagger}(A) \triangleq J^{-1}\left(A-A^{\mathrm{T}}\right)
$$

The maps $J^{-1}$ and $J^{\dagger}$ have distinct domains, and must not be confused. On the other hand, we will be sloppy and not distinguish between the version of the linear map $J$ whose range is skew $(3)$ and the version whose range is $\mathbb{R}^{3 \times 3}$.

The natural inner product on the vector space $\mathbb{R}^{3 \times 3}$ is

$$
\left(R_{1} \mid R_{2}\right) \triangleq \frac{1}{2} \operatorname{tr}\left\{R_{1} R_{2}^{\mathrm{T}}\right\}
$$

Direct computation reveals that $J$ is an isometry between $\mathbb{R}^{3}$ with its Euclidean norm, $\|w\|^{2}=$ $w^{\mathrm{T}} w$, and skew(3) with the norm corresponding to this inner product. Note, as well, that sym(3) is the orthogonal complement of skew(3) with respect to this inner product. Finally, the norm associated with $(\cdot \mid \cdot)$ defines a metric on the group $S O(3)$,

$$
\rho\left(R_{1}, R_{2}\right) \triangleq \xi^{-1} \circ\left(R_{1}-R_{2} \mid \dot{R_{1}}-R_{2}\right)
$$

after composition with a suitable comparison function [18], ${ }^{6}$

$$
\xi \in \mathcal{K}_{\infty}[[0, \pi],[0,4]]: \chi \mapsto 2(1-\cos \chi)
$$

On any Lie group, we may take the differential of left (or right) inverse translation and this is the canonical means of identifying left (or right) invariant vector fields with the Lie algebra. Thus,

$$
T_{R} S O(3)=\left\{R J(w) \in \mathbb{R}^{3 \times 3}: w \in \mathbb{R}^{3}\right\}
$$

is identified once and for all with $\operatorname{so}(3)=\operatorname{skew}(3) \approx \mathbb{R}^{3}$, and we may take the tangent bundle to be the cross product

$$
T S O(3) \approx S O(3) \times \mathbb{R}^{3} \text {. }
$$

As sketched in Section 2.1, a mechanical control system arises from the choice of a kinetic energy. If $M \in$ sym $^{+}(3)$, a positive definite symmetric matrix, is the moment of inertia matrix of the rigid body then the kinetic energy at a phase, $v=(R, r) \in T S O(3)$, is

$$
\kappa(v)=\langle v \mid v\rangle \triangleq(J(r) R \mid J(r) R M)
$$

This leads to a mechanical control system, $\Sigma$, whose internal dynamics may be expressed in body coordinates as

$$
f_{\Sigma}\left(v_{u}\right) \triangleq\left[\begin{array}{c}
R J(r) \\
M^{-1}[u-J(r) M r]
\end{array}\right] .
$$

\footnotetext{
${ }^{6}$ The comparison functions, the group $\mathcal{K}_{r}\left(\mathcal{I}_{1}, \mathcal{I}_{2}\right)$, of monotone increasing $C^{r}$ diffeomorphisms between two real intervals appears extensively in the engineering stability literature [9]. Some properties are reviewed in [18, 24].
} 
For the present application, we have assumed the à priori designation of a desired "reference trajectory", $v_{d}: \mathbb{R} \rightarrow T \mathcal{J}$, which is "second order." That is to say, if $v_{d}(t)=(D, d)(t)$, then $\dot{D} \equiv D J(d)$. Now if $v=(A, a)$ denotes the actual trajectory of the rigid body, we will find it useful to consider the "error coordinate system" obtained via left translation by $v_{d}$,

$$
v_{e}=(E, e) \triangleq L_{v_{d}} v=\left(D^{\mathrm{T}} A, a-A^{\mathrm{T}} D d\right),
$$

preserving the second order property, $\dot{E}(t)=E J(e(t))$.

\subsubsection{A Navigation Function on $S O(3)$}

We are now ready to search for a potential function on $S O(3)$ whose lift into the physical Lagrangian system of the actuated rigid body will define almost globally asymptotically stable error dynamics. According to Theorem 2, we need merely ensure that $\varphi$ is a navigation function. Since the configuration space has an empty boundary, this amounts to finding a Morse function with a unique minimum on $S O(3)$.

In a very nice report, Meyer [29] attempted to generalize PD techniques to the global control of spacecraft attitude. His point of view is very close to the spirit of this paper, and, in some sense, this application might be seen as a continuation and extension of that earlier work. Meyer chose for his potential law on $S O(3)$ the distance from a reference point measured by the natural metric, $\rho$, itself. This will not suffice for the present purposes since, the gradient of the distance function is necessarily undefined on its "cut locus" (the set of points whose minimal geodesic to a reference point is not unique) - an embedding of real projective two-space in $S O(3)$ in the present case. Intuitively, this is clear since $\rho$ is a composition with the trace function on skew(3). The latter is unfortunately not a Morse function since it has a critical point at every symmetric rotation: the symmetric rotations - an embedding of real projective two-space, $\mathbb{R P}^{2}$, in $S O(3)$ [7] - comprise a connected set; the critical points are not isolated. Instead we will use a "modified trace" function according to the following result of Marsden and collaborators.

Lemma 4.1 (Chillingworth, Marsden, and Wan [5] ) If $P \in \operatorname{sym}(3)$ has distinct eigenvalues, $\pi_{1}, \pi_{2}, \pi_{3}$, and

$$
\left(\pi_{1}+\pi_{2}\right)\left(\pi_{1}+\pi_{3}\right)\left(\pi_{3}+\pi_{2}\right) \neq 0,
$$

then there are exactly four rotations, $R \in S O(3)$ at which $P R \in \operatorname{sym}(3)$. These are exactly the critical points of

$$
(P \mid R)=\operatorname{tr}\{P R\}
$$

with Morse index specified by the number of positive eigenvalues of

$$
\operatorname{tr}\{R P\} I-P R
$$

We are thus led to define as a navigation function on $S O(3)$

$$
\varphi(R) \triangleq \frac{1}{\pi^{\prime}} \operatorname{tr}\{P(I-R)\}=\frac{1}{\pi^{\prime}}(P \mid I-R),
$$


where the factor involving $\pi^{\prime} \triangleq \pi_{2}+\pi_{3}-\pi_{1}$ is added to keep the image in the interval $[0,1]$ (assuming that $\pi_{1}<\pi_{2}<\pi_{3}$ ). If $P \in$ sym $^{+}(3)$, a positive definite symmetric matrix, which we now further assume, then the eigenvalue assumptions of the previous Lemma are assured. Since $d \varphi$ is a scalar multiple of Marsden's modified trace, $\varphi$ is also a Morse function with four critical points specified in the same fashion. Moreover, $\varphi$ takes its values on $\overline{\mathbb{R}^{\dagger}}$, vanishing only at $R=I$. Thus, $\varphi$ is indeed a navigation function. In fact, it is the best we can find since any Morse function on $S O(3)$ must have at least four critical points according to the LusternikSchnirelmann theorem [41, p. 92]. Moreover, it is certainly algebraic so that its differential one-form - the critical ingredient of the feedback law, $g(11)$ - may be readily computed [18] as

$$
d \varphi=2 J^{\dagger}(P R)
$$

According to Proposition 2.1 together with Lemma 4.1 the negative flow of the gradient vector field resulting from the kinetic energy metric,

$$
\operatorname{grad} \varphi=2 M^{-1} J^{\dagger}(P R),
$$

takes all points of $S O(3)$ to one of four symmetric rotations - the identity, and the three orientations which are " $180^{\circ}$ away" along the $x, y, z$ axes - and all points excepting a nowhere dense set to the identity.

\subsubsection{Inverse Dynamics}

In the linear time invariant setting, inverse dynamics amounts to the use of a precompensator to make the errors between the plant state and reference derivatives satisfy an asymptotically stable linear time invariant dynamical system. Entirely analogously in this setting, we will use the navigation function presented above to achieve an "almost global" asymptotically stable dynamical error system via the feedback law, $g(11)$, applied to the error coordinates, $v_{e}$. We then pre-filter the reference signal, $(D, d)(t)$, so that the error dynamics fall within the class of dissipative mechanical systems, $f_{\Delta}$, and apply Theorem 2 directly.

For example, in one degree of freedom, $\mathcal{J}=\mathbb{R}$, a point with unit mass gives rise to a kinetic energy metric given by the identification map, $M=1$, between the tangent and cotangent space over each configuration. If an arbitrary bidirectional force, $u$, can be imposed upon the point mass then our mechanical control system is the familiar double integrator,

$$
f_{\Sigma}\left(v_{u}\right) \triangleq\left[\begin{array}{l}
0 \\
u
\end{array}\right]
$$

Any Hook's Law spring potential, $\varphi \triangleq \frac{1}{2} K_{1} q^{2}$, (where $K_{1} \in \mathbb{R}^{+}$) in conjunction with a Rayleigh damper, $G_{d}(q, \dot{q}) \triangleq K_{2} \dot{q}$, (where $K_{2} \in \mathbb{R}^{+}$as well) defines a globally asymptotically stable closed loop system on $T \mathcal{J}=\mathbb{R}^{2}$,

$$
f_{\Delta}(v) \triangleq\left[\begin{array}{cc}
0 & 1 \\
-K_{1} & -K_{2}
\end{array}\right] v
$$


resulting from the feedback law

$$
g(q, \dot{q}) \triangleq K_{2} \dot{q}+K_{1} q
$$

given by (11). Given a particular reference trajectory, $v_{d}=(r, \dot{r})$, and an error coordinate system again defined via left translation (identical, of course, to right translation since $\mathbb{R}^{2}$ is an Abelian Lie group)

$$
v_{e}=(e, \dot{e}) \triangleq L_{v_{d}} v=(q-r, \dot{q}-\dot{r}),
$$

we may now servo on the error, $g\left(v_{e}\right)=K_{2} \dot{e}+K_{1} e$ and pre-filtering through the inverse of the closed loop plant,

$$
u \triangleq g\left(v_{e}\right)+\ddot{r}-K_{1} r-K_{2} \dot{r} .
$$

This results in globally asymptotically stable error dynamics,

$$
\dot{v}_{e}=f_{\Delta}\left(v_{e}\right)
$$

on $T \mathcal{J}$. We now implement a nonlinear version of this scheme in the present setting.

In place of the familiar Hooks's Law potential, we will substitute the navigation function, $\varphi$ (15). Taking an arbitrary positive definite symmetric matrix, $K_{2} \in S p(3)$, we will use Rayleigh damping, $G_{d}(R, r) \triangleq K_{2} r$, for the sake of simplicity. The feedback law (11) applied to the error coordinates is now computed as

$$
g(E, e)=K_{2} e+2 J^{\dagger}(P E)
$$

Note that

$$
\begin{aligned}
\dot{e} & =\dot{a}-E^{\mathrm{T}} \dot{d}-\left[E J\left(a-E^{\mathrm{T}} d\right)\right]^{\mathrm{T}} d \\
& =\dot{a}-E^{\mathrm{T}} \dot{d}+J(a) E^{\mathrm{T}} d .
\end{aligned}
$$

Thus, if we apply error feedback and build an appropriate pre-filter for the reference, $v_{d}=(D, d)$,

$$
\begin{aligned}
u \triangleq M & \left(E^{\mathrm{T}} \dot{d}-J(a) E^{\mathrm{T}} d\right) \\
& +J(a) M E^{\mathrm{T}} d+J\left(E^{\mathrm{T}} d\right) M e+g\left(v_{e}\right)
\end{aligned}
$$

the closed loop angular aceleration will be given as

$$
\dot{a}=E^{\mathrm{T}} \dot{d}-J(a) E^{\mathrm{T}} d-M^{-1}\left[J(e) M e+K_{2} e+\operatorname{grad} \varphi(E)\right],
$$

or, in the "error coordinate system" of phase space, $T S O(3)=S O(3) \times \mathbb{R}^{3}$,

$$
\begin{aligned}
\dot{E} & =E J(e) \\
\dot{e} & =-M^{-1}\left[J(e) M e+K_{2} e+\operatorname{grad} \varphi(E)\right] .
\end{aligned}
$$

Equation (17) is a dissipative mechanical system, $\Delta$ based upon the navigation function (15). Theorem 2 applies directly using information about the critical points of $\varphi$ supplied in Lemma 4.1. 
Theorem 4 All trajectories of (17) tend towand one of the four critical points of $\varphi . A$ dense open set of initial conditions has its limit set at the desired point, $(E, e)=(I, 0)$.

The satellite asymptotically attains the desired attitude trajectory, $D(t)$, except from a set of initial conditions of zero measure in the phase space.

\subsection{Robot Navigation}

Consider the following problem in robotics. A kinematic chain - a sequence of mutually constrained actuated rigid bodies - is allowed to move in a cluttered workspace. Contained within the joint space - an analytic manifold which forms the configuration space of the kinematic chain - is the free space, $\mathcal{F}$ - the set of all configurations which do not involve intersection with any of the "obstacles" cluttering the workspace. Given any interior "destination point," $q_{d} \in \stackrel{\circ}{\mathcal{F}}$, to which it is desired to move the robot, find a curve in $\mathcal{F}$ from an arbitrary initial point to the desired destination.

The purely geometric problem of constructing a path between two points in a space obstructed by sets with arbitrary polynomial boundary (given perfect information) has already been completely solved [40]. Moreover, a much more efficient solution has recently been offered for this class of problems as well [?]. The motivation for the present direction of inquiry (beyond its apparent academic interest) is the desire to incorporate explicitly aspects of the control problem - the construction of feedback compensators for a well characterized class of dynamical systems in the presence of well characterized constraints - in the planning phase of robot navigation problems. That is, the geometrical "find path" problem is generalized to the search for a family of paths in $\mathcal{F}$ (the one-parameter group of the gradient flow), which provides a feedback control law for the physical robot as well. It is clear from Theorem 2 that the construction of a navigation function on the freespace provides a solution to this problem.

\subsubsection{The Mechanical Control System}

The configuration space of a rigid robot with $n$ moving joints is generally taken to lie within the cross product of a torus and a cartesian space $\mathcal{J} \subset T^{n-k} \times \mathbb{R}^{k}$, where $k$ is number of "sliding joints" and $n-k$ is the number of "revolute joints" $[23,6]$, its boundary generally arising from physical limits on the range of motion of each joint. There is a "kinematic map," $k_{i}: \mathcal{J} \rightarrow \mathbb{R}^{3} \times S O(3)$, which expresses the physical location and orientation of a distinguished frame of reference in the $i^{\text {th }}$ constituent rigid body of the robot robot as a function of the position in configuration space $-k_{i}$ is a polynomial in transcendental functions of the generalized coordinates of $\mathcal{J}$. The "workspace," $\mathcal{W}$, lies within the $n$-fold cross product of this Euclidean group with itself, and represents the placements of the robot which do not intersect physical obstacles. The freespace, $\mathcal{F} \subset \mathcal{J}$ results after removing from $\mathcal{J}$ those configurations which involve any self-interesection or intersection with the obstacles. 
The kinetic energy is determined by summing up the contribution of each of the robot's constituent rigid bodies. Let $\left\{M_{i}\right\}_{i=1} n$ be the moment of inertia matrices of these constituent rigid bodies. In the appropriate local coordinates at some point $q \in \mathcal{J}$, the kinetic energy morphism, is given by [23]

$$
M(q) \triangleq \sum_{i=1}^{n}\left[\left[D k_{i}\right](q)\right]^{\mathrm{T}} M_{i}\left[D k_{i}\right](q)
$$

so that given any phase, $v=(q, x)$,

$$
\kappa(q, x)=\frac{1}{2} x^{\mathrm{T}} M(q) x \triangleq \frac{1}{2}\langle v \mid v\rangle
$$

Perhaps the most difficult aspect of the "generalized piano mover's problem" [40] is a precise determination of the freespace, $\mathcal{F}$, from information about the robot's consituent rigid bodies and the obstacles in the world. We will presume that this information has been furnished in the form of an implicit representation for each obstacle boundary. In particular, we will consider progressively more complicated versions of the freespace, and present analytic algebraic navigation functions for arbitrary destinations. These constructions solve the piano mover's "findpath" problem (for almost every initial configuration) via the resulting gradient, $\Gamma$ on $\mathcal{F}$ according to Proposition 2.1. Moreover, they solve the piano mover's "find controller" problem (for almost every initial configuration in the bounded energy set, $\mathcal{E}^{1} \subseteq T \mathcal{F}$ according to Theorem 2 .

\subsubsection{Navigation Functions on Euclidean Sphere Worlds}

A "Euclidean sphere world" is a compact connected subset of $\mathbf{E}^{n}$ whose boundary is the disjoint union of a finite number, say $M+1$, of $(n-1)$-spheres. We suppose that perfect information about this space has been furnished in the form of $M+1$ center points $\left\{q_{i}\right\}_{i=0}^{M}$ and radii $\left\{\rho_{i}\right\}_{i=0}^{M}$ for each of the bounding spheres. In our previous work [25], we have shown how to use this information to build a navigation function on the particular sphere world, $\mathcal{M}$, considered as a simple freespace.

The proposed navigation function, $\varphi: \mathcal{M} \rightarrow[0,1]$, is a composition of three functions:

$$
\varphi \triangleq \sigma_{d} \circ \sigma \circ \hat{\varphi}
$$

The function $\hat{\varphi}$ is polar, almost everywhere Morse and analytic, it attains a uniform height on $\partial \mathcal{M}$ by blowing up there. Its image is "squashed" by the diffeomorphism, $\sigma$, of $[0, \infty)$ into $[0,1]$, where

$$
\sigma(x) \triangleq \frac{x}{1+x}
$$

resulting in a polar, admissible, and analytic function which is non-degenerate on $\mathcal{M}$ except at one point - the destination. This last flaw is repaired by $\sigma_{d}$.

We distinguish between "good" and "bad" subsets of $\mathcal{M}$. When a point belongs to the "good" set, we expect the negative gradient lines to lead to it (here it is just $\left\{q_{d}\right\}$ ). The "bad" subset 
includes all the boundary points of the free space, and we expect the cost at such a point to be high. Let $\gamma$ and $\beta$ denote analytic real valued maps whose zero-levels, i.e. $\gamma^{-1}(0), \beta^{-1}(0)$, are respectively, the "good" and "bad" sets. We define $\hat{\varphi}$ to be,

$$
\hat{\varphi} \triangleq \frac{\gamma}{\beta}
$$

where $\gamma: \mathcal{M} \rightarrow[0, \infty)$ is

$$
\gamma \triangleq \gamma_{d}^{k} \quad k \in \mathbb{N} ; \quad \gamma_{d} \triangleq\left\|q-q_{d}\right\|^{2}
$$

and $\beta: \mathcal{M} \rightarrow[0, \infty)$ is,

$$
\beta \triangleq \Pi_{i=0}^{M} \beta_{i}
$$

where

$$
\beta_{0} \triangleq \rho_{0}^{2}-\|q\|^{2} ; \beta_{j} \triangleq\left\|q-q_{j}\right\|^{2}-\rho_{j}^{2} j=1 \ldots M .
$$

Due to the parameter $k$ in $\hat{\varphi}$, the destination point is a degenerate critical point. To counteract this effect, the "distortion" $\sigma_{d}:[0,1] \rightarrow[0,1]$

$$
\sigma_{d}(x) \triangleq(x)^{\frac{1}{k}}, k \in \mathbb{N}
$$

is introduced, to change $q_{d}$ to a non-degenerate critical point.

Theorem 5 ( [25]) If the free space, $\mathcal{M}$, is a Euclidean sphere world then there exists a positive integer $N$ such that for every $k \geq N$, for any finite number of obstacles, and for any destination point in the interior of $\mathcal{M}$,

$$
\varphi=\sigma_{d} \circ \sigma \circ \hat{\varphi}=\left(\frac{\gamma^{k}}{\gamma^{k}+\beta}\right)^{\frac{1}{k}}
$$

is a navigation function on $\mathcal{M}$.

In the proof of this theorem (which comprises the central contribution of [25]) a constructive formula for $N$ is given with the "schematic" form,

$$
N=\max _{\left\{\mathcal{O}_{i}\right\}_{i=0}^{M}} N_{i}\left(q_{d},\left[\begin{array}{c}
q_{0} \\
\rho_{0}
\end{array}\right], \ldots\left[\begin{array}{c}
q_{M} \\
\rho_{M}
\end{array}\right]\right),
$$

where $\mathcal{O}_{i}$ is the $i^{\text {th }}$ obstacle. The functions $N_{i}$ are given explicitly in an appendix of [25].

\subsubsection{Navigation Functions Induced by Diffeomorphism}

The Euclidean sphere world, of course, corresponds to a rather simplistic view of freespace. Fortunately, the navigation properties defined in Section 3.3 are invariant diffeomorphism, as the following result makes clear. 
Proposition $4.2([25])$ Let $\varphi: \mathcal{M} \rightarrow[0,1]$ be a navigation function on $\mathcal{M}$, and $h: \mathcal{F} \rightarrow \mathcal{M}$ be a diffeomorphism. Then

$$
\tilde{\varphi} \triangleq \varphi \circ h,
$$

is a navigation function on $\mathcal{F}$.

This result suggests that we might consider the Euclidean sphere world as a a "model space" used to induce navigation functions on more interesting "real spaces" in its analytic diffeomorphism class. The problem of constructing a navigation function on a member of this class reduces to the construction of an analytic diffeomorphism from this space onto its model.

Our constructive results to date encompass the class of "6tar worlds." A star shaped set is a diffeomorph of a Euclidean $n$-disk, $\mathcal{D}^{n}$ possessed of a distinguished interior center point from which all rays intersect its boundary in a unique point. A star world is a compact connected subset of $\mathbf{E}^{n}$ whose boundary is the disjoint union of a finite number of star shaped set boundaries. We now suppose the availability of an implicit representation for each boundary component, $\left\{\beta_{j}\right\}_{j=0}^{M}$, where $\beta_{j} \in C^{\omega}[\mathcal{F}, \mathbb{R}]$ and

$$
\partial \mathcal{F} \subseteq \bigcup_{j=0}^{M} \beta_{j}^{-1}[0]
$$

as well as the obstacle center points, $\left\{q_{j}\right\}_{j=0}^{M}$. Further geometric information required in the construction to follow is detailed in the chief reference for this work [37]. A suitable Euclidean sphere world model, $\mathcal{M}$, is explicitly constructed from this data. That is, we determine $\left(p_{j}, \rho_{j}\right)$, the center and radius of a model $j^{\text {th }}$ sphere, according to the center and minimum "radius" (the minimal distance from $q_{j}$ to the $j^{\text {th }}$ obstacle) of the $j^{\text {th }}$ star shaped obstacle. This in turn determines the model space "obstacle functions", $\left\{\hat{\beta}_{j}\right\}$ as well as the navigation function on $\mathcal{M}, \hat{\varphi}$, as described above.

A transformation, $h: \mathcal{M} \rightarrow \mathcal{F}$, may now be constructed in terms of the given star world and the derived model sphere world geometrical parameters as follows. Denote the " $j^{\text {th }}$ omitted product", $\Pi_{j=0}^{M} \beta_{j}$ as $\bar{\beta}_{j}$. The " $j^{t h}$ analytic switch", $\sigma_{j} \in C^{\omega}[\mathcal{F}, \mathbb{R}]$,

$$
\sigma_{j}(q, \lambda) \triangleq \frac{x}{x+\lambda} \circ \frac{\gamma_{d} \bar{\beta}_{j}}{\bar{\beta}_{j}}=\frac{\gamma_{d} \bar{\beta}_{j} b}{\gamma_{d} \bar{\beta}_{j} b+\lambda \bar{\beta}_{j}},
$$

(where $\lambda$ is a positive constant) attains the value one on the $j^{\text {th }}$ boundary and the value zero on every other boundary component of $\mathcal{F}$. The " $j^{\text {th }}$ star set deforming factor", $\nu_{j} \in C^{\omega}[\mathcal{F}, \mathbb{R}]$,

$$
\nu_{j}(q) \triangleq \rho_{j} \frac{1+\bar{\beta}_{j}(q)}{\left\|q-q_{j}\right\|},
$$

scales the ray starting at the center point of the $j^{\text {th }}$ obstacle, $q_{j}$, through its unique intersection with that obstacle's boundary in such a way that $q$ is mapped to the corresponding point on the $j^{\text {th }}$ model obstacle - a suitable sphere. The overall effect is that the complicated star shaped obstacle is is "deformed along the rays" originating at its center point on to the corresponding sphere in model space. 
Definition 2 The star world transformation, $h_{\lambda}$, is a member of the one-parameter family of analytic maps from an open neighborhood, $\tilde{\mathcal{F}} \subset \mathbf{E}^{n}$, containing $\mathcal{F}$, into $\mathbf{E}^{n}$, defined by

$$
h_{\lambda}(q) \triangleq \sum_{j=0}^{M} \sigma_{j}(q, \lambda)\left[\nu_{j}(q) \cdot\left(q-q_{j}\right)+p_{j}\right]+\sigma_{d}(q, \lambda)\left[\left(q-q_{d}\right)+p_{d}\right]
$$

where $\sigma_{j}$ is the $j^{\text {th }}$ analytic switch, $\sigma_{d}$ is defined by

$$
\sigma_{d} \triangleq 1-\sum_{j=0}^{M} \sigma_{j}
$$

and $\nu_{j}$ is the $j^{\text {th }}$ star set deforming factor.

The "switches", make $h$ look like the $j^{\text {th }}$ deforming factor in the vicinity of the $j^{\text {th }}$ obstacle, and like the identity map away from all the obstacle boundaries. With some further geometric computation we are able to prove the following.

Theorem 6 ( [37] ) For any valid star world, $\mathcal{F}$, there exists a suitable model sphere world $\mathcal{M}$, and a positive constant $\Lambda$, such that if $\lambda \geq \Lambda$, then

$$
h_{\lambda}: \mathcal{F} \rightarrow \mathcal{M} \text {, }
$$

is an analytic diffeomorphism.

Thus, if $\varphi$ is a navigation function on $\mathcal{M}$, the construction of $h_{\lambda}$ automatically induces a navigation function on $\mathcal{F}$ via composition, $\tilde{\varphi} \triangleq \varphi \circ h_{\lambda}$, according to Proposition 4.2.

This family of transformations, mapping any star world onto the corresponding sphere world, induces navigation functions on a much larger class than the original sphere worlds, thus advancing our program of research toward the goal of developing "geometric expressiveness" rich enough for navigation amidst real world obstacles. 


\section{Conclusion}

In recent years there has been a general resurgence of interest in the solution of abstract goals which are expressed by means of a constrained optimization problem. Extending well beyond the increasing body of robotics research $[32,16,35]$, this point of view informs the recent activity in neural network research [43], and simulated annealing methods of VLSI design [17], as well. The appeal of cost functions, of course, is that they lead to gradient vector fields: the optimization problem is "solved by integrating" the gradient dynamics on a "network" of digital computers. Gradient vector fields, in contrast to most other classes of nonlinear dynamical systems, are known to possess simple limit sets - the extrema of the cost function. The central result of this paper, Theorem 2, shows that the dynamics arising from the natural motion of appropriately compensated mechanical systems are capable of "integrating out" the limit set of a gradient system as well. Thus the mechanical plant may itself be used as a "second order" analog computer to solve problems encoded via cost functions.

Although Theorem 1 has been known for more than a century and the present extension, Theorem 2, involves the most elementary application of qualitative dynamical systems theory, this work represents (to the best of the author's knowledge) the first systematic use of of total energy for synthesizing desirable global properties in closed loop mechanical control systems. In particular, the notion of navigation functions and demonstration, Theorem 3 , that they exist on any reasonable configuration space is entirely new. Their utility is demonstrated by the new global results for satellite attitude tracking summarized in Theorem 4 , and for our incipient program of research in robot osbtacle avoidance, summarized by Theorem 5 and Theorem 6 .

There are at least two important failings of the theory presented here. The first arises from the "flawed" nature of total energy, $\eta$, when considered as a Lyapunov function: its derivative along the motions of a dissipative mechanical system, $\Delta$, vanishes on the entire zero section of the phase space, $T \mathcal{J}$. Recent work [24] has resulted in a family of modified total energy functions whose derivatives vanish only on the equilibrium states of $\Delta$ : thus the full power of Lyapunov theory as a tool for studying robustness [20], convergence rates [22], and adaptive capabilities [19] of dissipative mechanical systems is now available. More problematic, Lyapunov analysis, itself, reveals little concerning transient properties of dynamical systems beyond (often crude) convergence rate estimates. While the phase portrait of a gradient system, $\Gamma$, is closely related to the level curves of the defining scalar map, $\varphi$, it is not at all clear how to "tune" the dissipative field, $f_{d}$, to obtain analogous behavior from the dissipative mechanical system, $\Delta$. Preliminary results on a theory of "damping" for $\Delta$ have been presented in [19]: more comprehensive research addressing this question is presently in progress. 


\section{Acknowledgements}

A number of conversations with R. Brockett, E. Rimon, and E. Sontag have been extremely helpful in preparing this manuscript.

\section{References}

[1] Ralph Abraham and Jerrold E. Marsden. Foundations of Mechanics. Benjamin/Cummings, Reading, MA, 1978.

[2] N. P. Bhatia and G. P. Szegö . Dynamical Systems: Stability Theory and Applications. Springer-Verlag, Berlin, 1967.

[3] R. W. Brockett. Control theory and analytical mechanics. In C. Martin and R. Hermann, editors, Proc. 1976 NASA Ames Research Center Conference on Geometric Control Theory, pages 1-49, Mathsci Press, 1977.

[4] N. G. Chetaev. The Stability of Motion. Pergammon, New York, 1961.

[5] D. R. J. Chillingworth, J. E. Marsden, and Y.H. Wan. Symmetry and bifurcation in threedimensional elasticity, Part I. Arch. Rat. Mech. Anal., 80(4):295-331, 1982.

[6] John J. Craig. Introduction to Robotics Mechanics and Control. Addison Wesley, Reading, MA, 1986.

[7] Theodore Frankel. Critical submanifolds and stiefel manifolds. In Stewart S. Cairns, editor, Differential and Combinatorial Topology, pages 37-54, Princeton University, Princeton, 1965.

[8] H. Goldstein. Classical Mechanics. Addison-Wesley, Reading, MA, 1980.

[9] Wolfgang Hahn. Stability of Motion. Springer-Verlag, New York, 1967.

[10] Morris W. Hirsch. Differential Topology. Springer-Verlag, NY, 1976.

[11] Morris W. Hirsch and Stephen Smale. Differential Equations, Dynamical Systems, and Linear Algebra. Academic Press, Inc., Orlando, Fla., 1974.

[12] Neville Hogan. Impedance control: an approach to manipulation. ASME Journal of Dynamics Systems, Measurement, and Control, 107:1-7, Mar 1985.

[13] T.A.W. Dwyer III. Exact nonlinear control of large angle rotational maneuvers. IEEE Transactions on Automatic Control, AC-29(9):769-774, 1984.

[14] R.E. Kalman and J.E. Bertram. "Control systems analysis and design via the 'second method' of Lyapunov". Journal of Basic Engineering, 371-392, June 1960. 
[15] O. Khatib and J.-F. Le Maitre. Dynamic control of manipulators operating in a complex environment. In Proceedings Third International CISM-IFToMM Symposium, pages 267282, Udine, Italy, Sep 1978.

[16] Oussama Khatib. Real time obstacle avoidance for manipulators and mobile robots. The International Journal of Robotics Research, 5(1):90-99, Spring 1986.

[17] S. Kirkpatrick, C. D. Gelatt, and M. P. Vecchi. Optimization by Simulated Annealing. Research Report RC 9355 (\#41093), IBM Thomas J. Watson Research Center, Yorktown Heights, NY, Apr 1982.

[18] D. E. Koditschek. Application of a new Lyapunov function to global adaptive attitude tracking. In Proc. 27th IEEE Conference on Decision and Control, pages 63-68, Austin, TX, Dec 1988.

[19] Daniel E. Koditschek. Adaptive techniques for mechanical systems. In Fifth Yale Workshop on Applications of Adaptive Systems Theory, pages 259-265, Center for Systems Science, Yale University, New Haven, CT, May 1987.

[20] Daniel E. Koditschek. High gain feedback and telerobotic tracking. In Workshop on Space Telerobotics, pages 355-363, Jet Propulsion Laboratory, California Institute of Technology, Pasadena, CA, Jan 1987.

[21] Daniel E. Koditschek. Natural motion for robot arms. In IEEE Proceedings 23rd Conference on Decision and Control, pages 733-735, Las Vegas, Dec 1984.

[22] Daniel E. Koditschek. Quadratic Lyapunov Functions for Mechanical Systems. Technical Report 8703, Center for Systems Science, Yale University, Mar 1987.

[23] Daniel E. Koditschek. Robot control systems. In Stuart Shapiro, editor, Encyclopedia of Artificial Intelligence, pages 902-923, John Wiley and Sons, Inc., 1987.

[24] Daniel E. Koditschek. Strict global lyapunov functions for mechanical systems. In Proc. American Control Conference, pages 1770-1775, American Automatic Control Council, Atlanta, GA., Jun 1988.

[25] Daniel E. Koditschek and Elon Rimon. Robot navigation functions on manifolds with boundary. Advances in Applied Mathematics, (to appear).

[26] J. L. LaGrange. Méchanique Analytique. Gauthier-Villars, Paris, 1788.

[27] J. P. Lasalle. The Stability of Dynamical Systems. Volume 25 of Regional Conference Series in Applied Mathematics, SIAM, Philadelphia, PA, 1976.

[28] A. M. Lyapunov. Problème Gén éral de la Stabilité du Mouvement. Princeton University, Princeton, NJ, 1949.

[29] George Meyer. Design and Global Analysis of Spacecraft Attitude Control Systems. NASA Technical Report TR R-361, Ames Research Center, Moffett Field, CA, Mar 1971. 
[30] J. Milnor. Lectures on the h-Cobordism Theorem. Princeton University Press, Princeton, NJ, 1965.

[31] John W. Milnor. Topology from the Differentiable Viewpoint. The University Press of Virginia, Charlottesville, Va., 1965.

[32] Fumio Miyazaki and S. Arimoto. Sensory feedback based on the artificial potential for robots. In Proceedings 9th IFAC, Budapest, Hungary, 1984.

[33] Marston Morse. The existence of polar non-degenerate functions on differentiable manifolds. Annals of Mathematics, 71(2):352-383, Mar 1959.

[34] Jacob Palis, Jr. and Welington de Melo. Geometric Theory of Dynamical Systems. SpringerVerlag, New York, 1982.

[35] V. V. Pavlov and A. N. Voronin. The method of potential functions for coding constraints of the external space in an intelligent mobile robot. Soviet Automatic Control, (6), 1984.

[36] Ralph Pringle, Jr. On the stability of a body with connected moving parts. AIAA, 4(8):1395-1404, Aug 1966.

[37] E. Rimon and D. E. Koditschek. The construction of analytic diffeomorphisms for exact robot navigation on sphere worlds. In Proc. IEEE International Conference on Robotics and Automation, (to appear), Arizona, May 1989.

[38] A. J. Van Der Schaft. Stabilization of Hamiltonian Systems. Memo 470, Technische Hogeschool Twente, Twente, Netherlands, Jan 1985.

[39] Jacob T. Schwartz and Micha Sharir. On the 'Piano Movers' problem. II. general techniques for computing topological properties of real algebraic manifolds. Advances in Applied Mathematics, 4:298-351, 1983.

[40] Jacob T. Schwartz and Micha Sharir. On the "Piano Movers" Problem I. The Case of a Two-Dimensional Rigid Polygonal Body Moving Amidst Polygonal Barriers. Technical Report 39, N.Y.U. Courant Institute Department of Computer Science, New York, 1981.

[41] H. Seifert and W. Threfall. Variationsrechnung im Grossen (Theorie von Marston Morse). Chelsea, New York, 1948.

[42] Morikazu Takegaki and Suguru Arimoto. A new feedback method for dynamic control of manipulators. ASME Journal of Dynamics Systems, Measurement, and Control, 102:119$125,1981$.

[43] David W. Tank and John J. Hopfield. Simple "Neural Optimization Networks": an a/d converter, signal decision circuit, and a linear programming circuit. IEEE Transactions on Circuits and Systems, CAS-33(5):533-541, May 1986.

[44] Sir W. Thompson and P. G. Tait. Treatise on Natural Philosophy. University of Cambridge Press, 1886, Cambridge. 